\title{
REDES, SERVIÇOS DE SAÚDE E CIDADES PEQUENAS NO TERRITÓRIO DO SISAL.
}

Géssica de Oliveira Santos ${ }^{1}$; Edinusia Carneiro Moreira Santos ${ }^{2}$

1. Bolsista PIBIC/FAPESP, Graduando em Geografia, Universidade Estadual de Feira de Santana, e-mail: geogessica@gmail.com

2. Orientador, Departamento de Ciências Humanas e Filosofia-DCHF, Universidade Estadual de Feira de Santana, email: nusiafs@hotmail.com

PALAVRAS-CHAVE: Serviços De Saúde; Redes; Cidades Pequenas.

\section{INTRODUÇÃO}

Com base em Webster, (2002), as redes de serviços foram constituídas com intuito de sistematizar os serviços de saúde para o benefício de populações em determinada área. Contudo, os serviços de saúde constituíram-se como um importante parâmetro para caracterizar a dinâmica espacial das cidades pequenas do Território do Sisal, diante dos serviços oferecidos do setor público e privado e a relação entre os fluxos e fixos na reorganização das Cidades Pequenas do Território do Sisal.

\section{MATERIAL E MÉTODOS OU METODOLOGIA (ou equivalente)}

A metodologia utilizada neste estudo tem caráter exploratório, na qual se trabalha com dados secundários, isto é, material que já foi publicado, e que se encontra disponível, em um ou vários canais de divulgação (BARROS, 1990).

- REVISÃO BIBLIOGRÁFICA

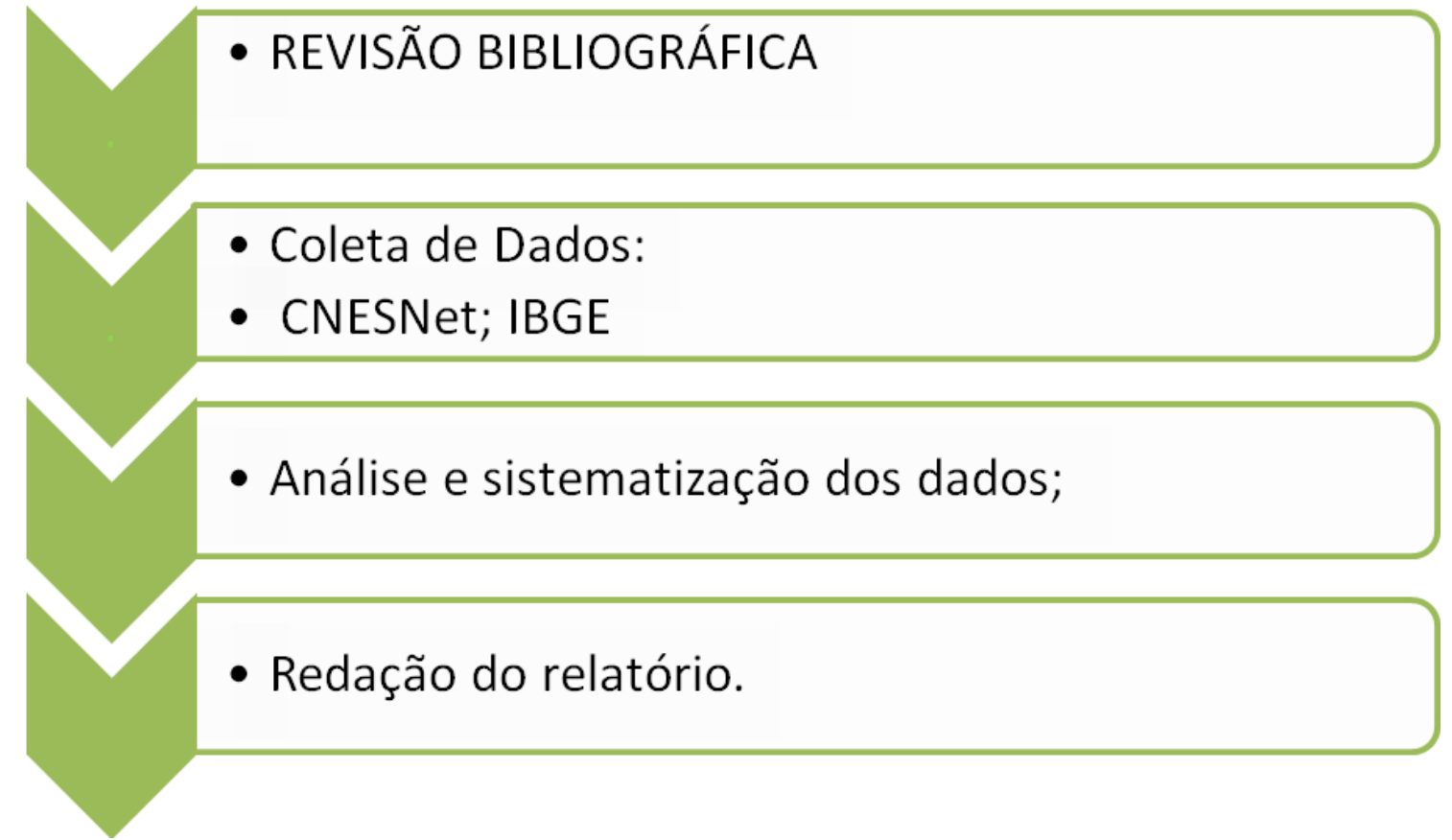

FONTE: Iniciação Científica, 2017

Elaboração: Géssica de Oliveira Santos 


\section{RESULTADOS E/OU DISCUSSÃO (ou Análise e discussão dos resultados)}

Tabela 1. Nível de complexidade dos Serviços de saúde existentes nas Cidades Pequenas no Território do Sisal.

\section{SERVIÇOS DE SAÚDE}

Posto de saúde

Centro de saúde/unidade básica

Policlínica

Unidade de vigilância em saúde

Central de regulação do acesso

Centro de apoio à saúde da família

Unidade mista

Hospital geral

Clinica/centro de especialidade

Unidade de apoio diagnose e terapia (sadt isolado)

Unidade móvel de nível pré-hospitalar da área de urgência

Unidade móvel terrestre

Central de notificação, captação e distribuição de órgãos estadual

Centro de atenção psicossocial

Laboratório de saúde pública

Pronto atendimento
NÍVEL DE COMPLEXIDADE

Legenda: São diversos tipos de unidades que compõe a rede do sistema de saúde público e privado que são assistenciais, como unidades básicas de saúde, consultórios, hospitais, clínicas, laboratórios, entre outros em que os níveis de complexidade dos serviços de saúde das Cidades Pequenas são articulados em níveis primário e secundário, estabelecendo pontos na rede e oferta de variados serviços de saúde.

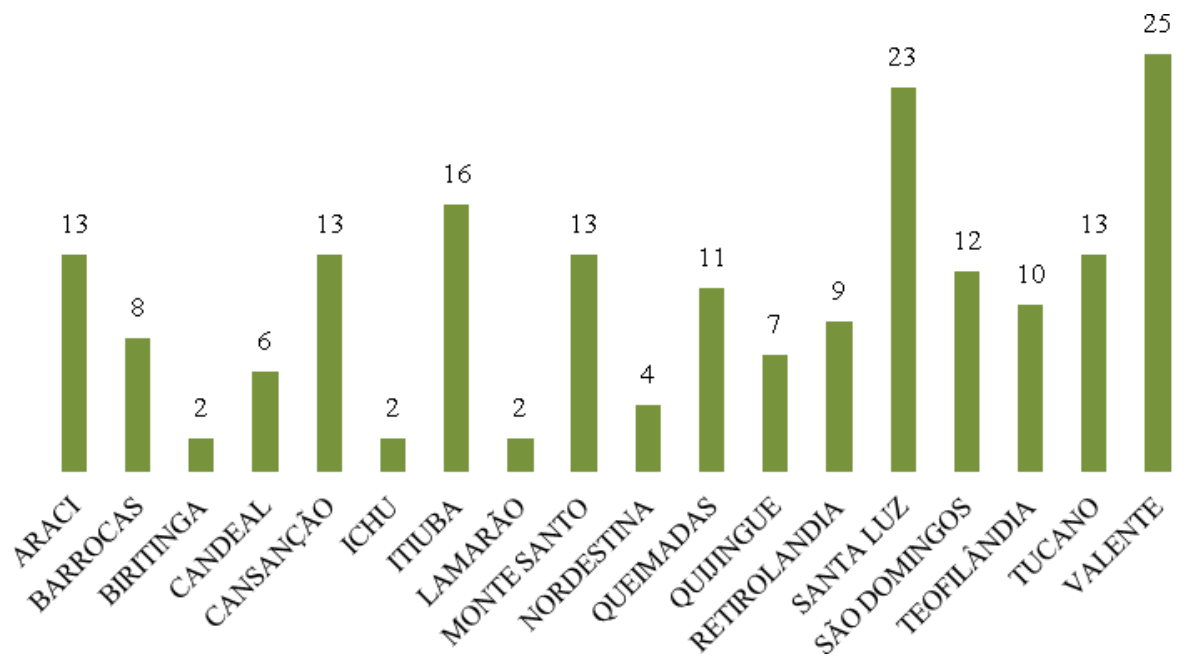

FIGURA 1: Relação da quantidade dos Serviços de saúde das Cidades Pequenas no Território do Sisal. LEGENDA: De acordo com a coleta dos dados secundários disponíveis no Cadastro Nacional de Estabelecimentos de Saúde (CNESNet), as Cidades Pequenas do Território do Sisal: Araci, Cansanção, 
Itiúba, Monte Santo, Queimadas, Santa Luz, São Domingos, Tucano e Valente, Barrocas, Biritinga, Candeal, Ichu, Lamarão, Nordestina, Quijingue, Retirolândia e Teofilândia, ao total existem cento e noventa e um (191) estabelecimentos de saúde, sendo eles público e privado.

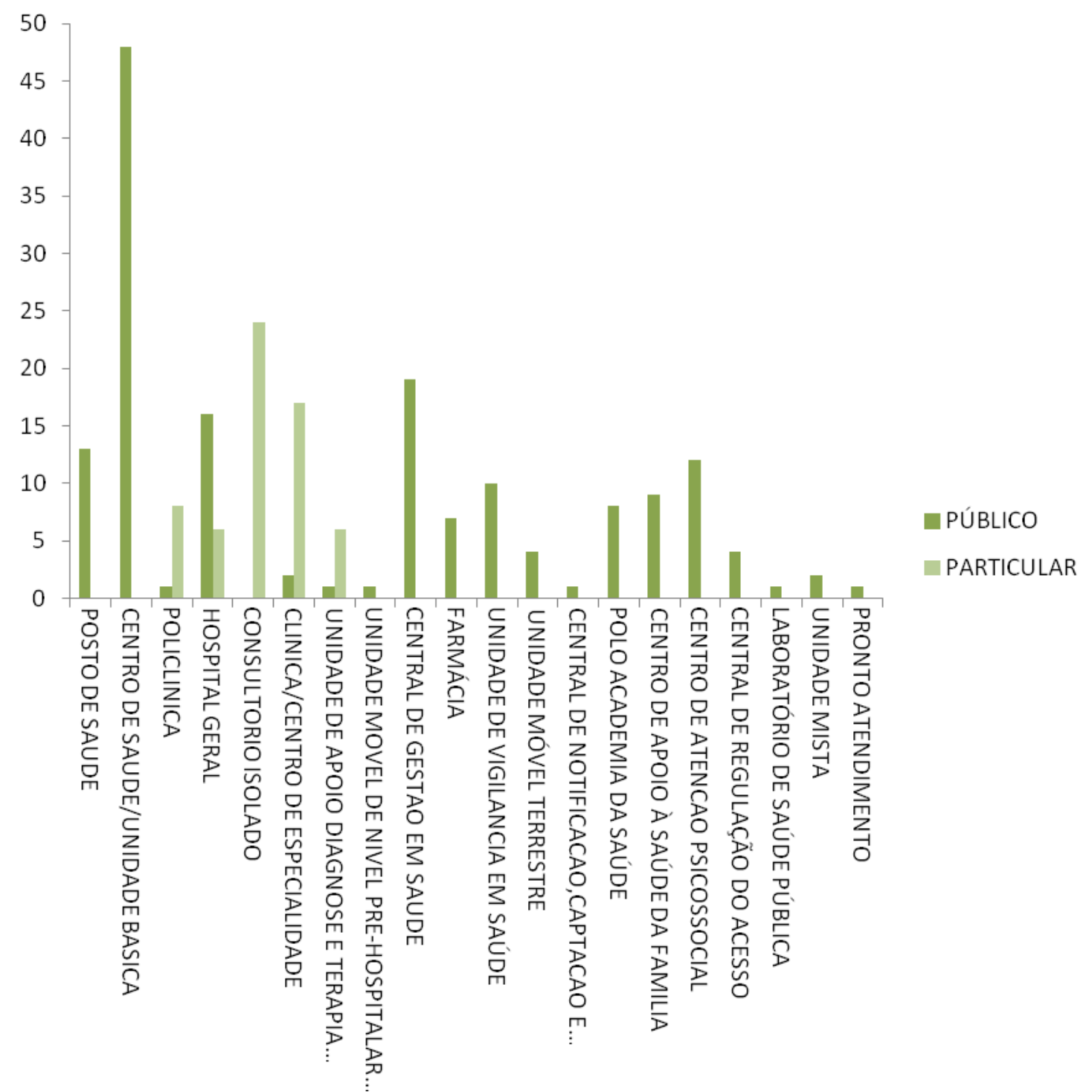

Figura 2: Oferta dos Serviços de Saúde pelo setor público e setor privado nas Cidades Pequenas no Território do Sisal.

Legenda: Consta-se de que a maioria dos serviços de saúde nas Cidades Pequenas no Território do Sisal é do setor público.

Nas cidades pequenas do Território do Sisal, verifica-se que não há uniformidade na distribuição dos serviços de saúde. Diante da coleta dos dados contatou-se que as cidades de Araci, Monte Santo, Tucano e Valente são as que mais possuem estabelecimentos de saúde principalmente do setor público. Com isso, os pacientes de outras Cidades Pequenas do Território do Sisal vão em busca desses serviços, impulsionando deslocamentos de pessoas em busca de serviços de saúde.

O serviço de saúde oferecido nas Cidades Pequenas do Território do Sisal é de forma desigual. As cidades que menos possuem estabelecimentos de saúde é Biritinga, Candeal, Ichu, Lamarão e Nordestina. Consequentemente os pacientes dessas cidades vão à busca de serviços de saúde em outras Cidades Pequenas. E com detalhe, essas cidades não possuem Central de Regulação do Acesso para que por meio do SUS possam ter acesso mais rápido ao atendimento nos serviços de saúde das outras cidades. 
Cada cidade pequena possui sua organização, estrutura e administração para atender as necessidades da população principalmente nos níveis primários, que são as unidades básicas de saúde, em que é o que mais se verifica entre as Cidades Pequenas.

A partir do que foi caracterizado, identificamos algumas cidades que podem ser agrupadas em função de possuírem características bem parecidas quanto aos estabelecimentos de saúde. Assim:

As cidades de Ichu, Lamarão, Candeal, Nordestina, Biritinga; possuem número reduzido e pequena variação nos tipos de estabelecimentos, predominando os públicos;

- Cansanção, Retirolândia, São Domingos, Barrocas, Monte Santo, Quijingue, Queimadas, Teofilândia; cidades que possuem um número relativo de estabelecimentos e variedade, mas que não estão não se aproxima das cidades no grupo em destaque.

- Valente, Tucano, Araci, Santa Luz e Itiúba; são cidades que se destacam em função da quantidade e da variedade de estabelecimentos de saúde.

\section{CONSIDERAÇÕES FINAIS (ou Conclusão)}

Diante da pesquisa realizada, os estabelecimentos públicos são a maioria dos Serviços de saúde das Cidades pequenas no Território do Sisal. Porém, algumas cidades pequenas possuem maior número de estabelecimento público e privado, onde contribuem com a dinâmica no Território do Sisal, na relação dos fixos e fluxos e na reorganização das Cidades Pequenas.

\section{REFERÊNCIAS}

BRASIL. MINISTÉRIO DA SAÚDE. DATASUS. Tipo de Estabelecimento. Disponível em: <http://tabnet.datasus.gov.br/cgi/cnes/tipo_estabelecimento.htm> Acesso em: 22 de Fevereiro 2017.

BRASIL. Conselho Nacional de Secretários de Saúde. A Atenção Primária e as Redes de Atenção à Saúde. Conselho Nacional de Secretários de Saúde. Brasília: CONASS, 2015. $127 \mathrm{p}$.

FARIA, R. V. A territorialização da atenção primária à saúde no sistema único de saúde e a construção de uma perspectiva de adequação dos serviços aos perfis do território. Hygeia, Florianopolis, v.9, n.16, p. 131 - 147, Jun/2013.

OLIVEIRA, N. R. C. UNIVERSIDADE FEDERAL DO MARANHÃO. UNA-SUS. Redes de Atenção à Saúde: a atenção à saúde organizada em redes. São Luís: EDUFMA, 2016. 54f.

PEREHOUSKEI, N. A. et al. A organização dos serviços de saúde na práxis territorial. Sociedade e Território, Natal, v. 27, nº 1, p. 101-116, 2015.

DIAS, L. C. Redes: emergência e organização. In: CASTRO, I. E.(Org) et al. Geografia: conceitos e temas. Rio de Janeiro: Bertrand Brasil, 1995. p.141 - 162. 